\title{
ENSEÑAR MINDFULNESS: \\ CONTEXTOS DE INSTRUCCIÓN Y PEDAGOGÍA
}

\section{TEACHING MINDFULNESS: \\ CONTEXTS OF TEACHING AND PEDAGOGY}

\author{
Ausiàs Cebolla \\ Universitat de València \\ Daniel Campos \\ Universitat Jaume I
}

Cómo referenciar este artículo/How to reference this article:

Campos, D. y Cebolla, A. (2016). Enseñar Mindfulness: Contextos de Instrucción y Pedagogía. Revista de Psicoterapia, 27(103), 103-118.

\begin{abstract}
Resumen
La aparición de mindfulness y su rápida diseminación en entornos clínicos obedece a diversas razones, siendo una de las más relevantes la relacionada con la pedagogía. La literatura cientifica ha propuesto un modelo de enseñanza de mindfulness basado en los mecanismos de eficacia, la configuración de nuevas demandas, y la aparición de diferentes contextos. El objetivo de este artículo es revisar por un lado los diferentes contextos de enseñanza de mindfulness (psicoeducativos, clínicos y espirituales), analizando las semejanzas y diferencias. A continuación se revisarán los mecanismos de eficacia (regulación de la atención, conciencia corporal, regulación emocional y cambios en la perspectiva del self) que fundamentan hacia donde hay que enfocar la instrucción, y finalmente se presentará el proceso de indagación basándonos en el trabajo realizado en la Terapia Cognitiva Basada en Mindfulness (TCBM), y que supone un elemento fundamental en la enseñanza de mindfulness. La pedagogía es un elemento comúnmente olvidado en los entrenamientos de instructores, y nos sitúa un nuevo horizonte en la investigación, generando una serie de retos de futuro que serán analizados.

Palabras clave: mindfulness, meditación, indagación, darse cuenta, metacognición
\end{abstract}

\begin{abstract}
The appearance of mindfulness and its rapid spread in clinical settings is due to various reasons, one of the most important being related to pedagogy. The scientific literature has proposed a model for teaching mindfulness based on the study of the efficacy mechanisms, new teaching demands, and the emergence of different contexts. The purpose of this paper is to review the different contexts of teaching mindfulness (psycho-educational, clinical and spiritual), analyzing the similarities and differences. The underlying mechanisms of efficacy (attention regulation, body awareness, emotional regulation and changes in the perspective of the self) that base where teachers need to focus instructions and finally the inquiry process will be presented based on the work done in Mindfulness Based Cognitive Therapy, that is a fundamental element in teaching mindfulness. Pedagogy is an element commonly forgotten in training of trainers, and sets up a new horizon in research, generating a series of challenges for the future that will be analyzed.
\end{abstract}

Keywords: Mindfulness, meditation, inquiry, awareness, metacognition

Fecha de recepción: 12/12/2015. Fecha de aceptación: 14/01/2016.

Correspondencia sobre este artículo:

E-mail: ausias.cebolla@uv.es

Dirección postal: Ausiàs Cebolla i Martí, Universitat de València, Avinguda Blasco Ibáñez, 10, 46010 València.

@) (C) 2016 Revista de Psicoterapia 
En el año 2014 la revista TIME publicó un número con una portada muy significativa, que rezaba "la revolución Mindfulness". Ciertamente esta portada capta muy bien todo lo que ha ido aconteciendo en los últimos años en la diseminación e investigación de mindfulness en occidente y que tiene efectivamente aspectos revolucionarios. Mindfulness ha pasado en pocos años de ser una práctica marginal asociada únicamente a contextos espirituales a estar omnipresente en las mejores revistas científicas y congresos del mundo, generando un impacto social que pocos podían predecir (los trabajos sobre mindfulness en nuestro país tienen ya más de una década, algunos de ellos se publicaron en un monográfico de la Revista de Psicoterapia dedicado a mindfulness: Cebolla y Miró, 2006; García, 2006; Miró, 2006; Pérez y Botella, 2006; Santamaría, Cebolla, Rodríguez y Miró, 2006; Simón, 2006).

Esta revolución se debe a varios factores; por un lado los estudios de eficacia han mostrado que puede ser una terapia altamente eficaz. Por ejemplo, recientemente la revista The Lancet, una de las mejores del mundo en medicina, publicaba un estudio mostrando la eficacia de la MBCT (Kuyken et al., 2015) para la prevención de recaídas en depresión. Por otro lado el segundo factor se debe a la demanda social en un mundo cada vez más tecnológico y multitarea. Las personas buscan desesperadamente espacios de calma, serenidad y autocuidado, lo que ha llevado a sentirse atraídos por las prácticas de tipo contemplativo. El tercer factor, al que pocos autores hacen referencia, tiene que ver con una revolución pedagógica. Paralela a la investigación de resultados ha habido una investigación sobre cómo transmitir estas enseñanzas que ha supuesto una forma innovadora y facilitadora de transmitirlas.

Mindfulness, como es ampliamente conocido, proviene del Budismo y aunque está presente en todas las prácticas espirituales es el Budismo el que ha sabido generar un método y un fundamento que ha atraído a los científicos occidentales. El reto al que se enfrenta mindfulness en occidente es variado. Por un lado el de ser investigado científicamente, lo cual plantea un riesgo de atomización y desvinculación de sus orígenes como muchos autores ya han destacado (Grossman, 2015); es decir mindfulness nunca ha sido utilizado para lo que se usa en occidente bienestar psicológico, tratamientos clínicos, etc. Por otro lado, la tradición no ha tenido que enfrentarse a un escenario como el hospitalario o clínico en el que hay personas que aprenden mindfulness sin tener una clara motivación, ni un deseo estructurado de aprendizaje; por ejemplo, una persona con depresión que simplemente quiere que alguien le ayude para sentirse mejor. Finalmente, también ha cambiado el método de diseminación, es decir ya no son maestros de meditación con largos años de experiencia los que enseñan mindfulness sino profesionales, sobre todo psicólogos y médicos, que a pesar de estar más o menos implicados en su práctica diaria no son estrictamente maestros de meditación, sino instructores de mindfulness. Además mindfulness también se está implementando de manera generalizada en entornos escolares y organizacionales, lo que supone una gran 
novedad. Estos nuevos contextos de instrucción hacen que los obstáculos o dificultades de la práctica que surgen (falta de motivación, mitos de la meditación, divagación mental, etc.) deban ser trabajados de manera coherente, con un sistema protocolizado y con una metodología pedagógica definida.

Todo esto, ha llevado a muchos autores a reflexionar sobre cómo se puede mejorar la enseñanza de esta herramienta adaptándola a estos nuevos retos, contextos y roles, con el objetivo final de llegar al mayor número de personas posible. El objetivo de este artículo es revisar lo que se ha investigado sobre pedagogía de mindfulness, los contextos y roles para su instrucción, mecanismos de eficacia y trabajo grupal, así como analizar posibles retos de futuro en la instrucción de mindfulness.

\section{El Instructor de Mindfulness y su Contexto}

Mindfulness es una herramienta que se puede utilizar por los menos en tres contextos de enseñanza diferentes (véase Tabla 1): (i) el espiritual, tradicionalmente el único contexto de enseñanza y con pedagogías basadas en las diferentes tradiciones de enseñanza que se han ido pasando de generación en generación, en algunos casos palabra por palabra. A este contexto, se le añaden dos nuevos, (ii) el contexto clínico (tratamiento de trastornos psicológicos, depresión, ansiedad, etc.) y (iii) el psicoeducativo (entorno escolar, organizacional o de crecimiento personal).

Cada uno de estos contextos, requiere profesionales con formaciones diferentes: en el caso del contexto espiritual, maestros de meditación; en el caso del contexto psicoeducativo, profesionales/instructores formados en mindfulness y expertos en el entorno donde lo vayan a aplicar (alguien del mundo de la empresa cuando se enseña en el ámbito organizacional, o un profesor en el ámbito escolar) y, finalmente, en el contexto clínico, terapeutas, psicólogos y médicos con formación en mindfulness. Además, cada contexto va acompañado de diferentes estructuras formativas y objetivos diferentes, que deben saber adaptar la pedagogía de mindfulness a los diferentes objetivos que se les plantea, desde mejorar el bienestar, hasta reducir sintomatología o constituir una vía de desarrollo espiritual. Esta versatilidad está relacionada con los mecanismos psicológicos que se activan en la práctica de mindfulness que hace que active procesos psicológicos profundamente nucleares.

Es fundamental que los instructores conozcan el contexto donde se mueven, ya que cualquier salto de contexto sin la formación adecuada puede ser catastrófico, desde maestros de meditación que trabajen en contextos clínicos a personas sin la preparación adecuada que trabajen en contextos espirituales.

A todo esto hay que añadir un punto de gran importancia. En los últimos años han surgido varias voces invitando a investigar si la práctica de mindfulness o meditación tiene efectos no esperados o indeseables. Los datos indican que estos efectos existen y que el porcentaje no es precisamente bajo (Cebolla, Demarzo, 


\section{Tabla 1}

Contextos de Enseñanza de Mindfulness

\begin{tabular}{|c|c|c|c|}
\hline & Contexto psicoeducativo & Contexto clínico & Contexto espiritual \\
\hline Objetivo & $\begin{array}{l}\text { Aumentar mindfulness rasgo, } \\
\text { bienestar psicológico, } \\
\text { felicidad, regulación } \\
\text { emocional, auto-compasión. }\end{array}$ & $\begin{array}{l}\text { Aumentar mindfulness } \\
\text { rasgo, reducción de } \\
\text { síntomas y de } \\
\text { vulnerabilidad cognitiva. }\end{array}$ & $\begin{array}{l}\text { Desapego, búsqueda de } \\
\text { la verdad, } \\
\text { desconstrucción del yo. }\end{array}$ \\
\hline Profesional & $\begin{array}{l}\text { Profesional formado en } \\
\text { mindfulness y experto en el } \\
\text { ámbito de enseñanza donde } \\
\text { se aplica. } \\
\text { (Instructor-participante) }\end{array}$ & $\begin{array}{l}\text { Psicólogo clínico, } \\
\text { psicólogo general } \\
\text { sanitario, psiquiatra. } \\
\text { (Terapeuta-paciente) }\end{array}$ & Maestro de meditación. \\
\hline Técnicas & $\begin{array}{l}\text { Mindfulness en la vida } \\
\text { cotidiana, meditaciones } \\
\text { sencillas. }\end{array}$ & $\begin{array}{l}\text { Mindfulness en la vida } \\
\text { cotidiana, meditaciones } \\
\text { sencillas. Apoyo a otras } \\
\text { intervenciones. }\end{array}$ & $\begin{array}{l}\text { Meditaciones más } \\
\text { complejas. }\end{array}$ \\
\hline $\begin{array}{l}\text { Duración de las } \\
\text { sesiones }\end{array}$ & Entre 20 y 40 minutos por día. & $\begin{array}{l}\text { Entre } 20 \text { y } 40 \text { minutos } \\
\text { por día. }\end{array}$ & Más flexibilidad. \\
\hline $\begin{array}{l}\text { Duración del } \\
\text { entrenamiento }\end{array}$ & Ocho semanas. & $\begin{array}{l}\text { Ocho semanas en formato } \\
\text { grupal e inespecífico en el } \\
\text { individual. }\end{array}$ & Depende de la tradición. \\
\hline Formato & Grupal & Grupal e individual & Grupal e individual \\
\hline
\end{tabular}

Martins, Soler y García-Campayo, en revisión; Shapiro, 1992). En estos casos el contexto es clave, ya que lo que en contexto se puede entender como un efecto no esperado (por ejemplo, un aumento de la tristeza en un contexto clínico) será visto como algo no deseable y por tanto se intentará paliar, mientras que en el contexto espiritual será visto como una parte fundamental del camino.

\section{¿Para qué Sirve Practicar Mindfulness?}

Entender bien los mecanismos psicológicos por los que mindfulness genera los cambios que genera es clave para su enseñanza, ya que es precisamente este conocimiento el que permite flexibilizar y diseñar intervenciones adaptadas al contexto de instrucción. Enseñar mindfulness para reducir estrés en un contexto organizacional (aprender a regular el estrés, mejorar la gestión del tiempo...) no tiene nada que ver con trabajar con mindfulness en un programa de prevención de recaídas en depresión (aumentar habilidades metacognitivas, regular la emoción, modificar de estrategias de afrontamiento desadaptativas...). Las técnicas pueden ser las mismas pero los objetivos y lo explorado en sesión es complemente diferente, y por tanto la pedagogía debe ser adaptada.

Dentro de los mecanismos de eficacia de mindfulness destaca el trabajo de Hölzel et al., (2011) en el que revisa los principales estudios llevados a cabo sobre meditación y mindfulness en los últimos años. Los autores reducen los mecanismos de actuación a cinco: (1) control atencional, (2) conciencia corporal, (3) regulación 
emocional, (4) revalorización y exposición y (5) un cambio en la perspectiva del self.

Estos mecanismos son una excelente plataforma para comprender la pedagogía de mindfulness ya que simulan de manera fehaciente todos los procesos activados en la práctica de mindfulness y que son precisamente los que lo hacen tan versátil. Los autores proponen que estos componentes suponen un proceso de mejora de la auto-regulación. Por tanto, el aprendizaje de mindfulness resultaría en una mejora de la capacidad de auto-regulación a distintos niveles. Los diferentes componentes interactúan entre sí y, además, entran en juego en distintos momentos de la práctica de mindfulness, como más adelante comentaremos. Trasladada a la práctica, esta propuesta teórica a partir de una excelente revisión podría seguirse como un plan de acción en la enseñanza de mindfulness (Tabla 2). A continuación se relata dicho plan por orden cronológico en términos pedagógicos (Cebolla, 2014).

\section{Tabla 2}

Mecanismos de Eficacia y Pedagogía de Mindfulness

\begin{tabular}{|c|c|c|c|c|}
\hline Mecanismos & $\begin{array}{l}\text { Instrucciones de la } \\
\text { práctica }\end{array}$ & $\begin{array}{l}\text { Ejemplos de } \\
\text { prácticas de } \\
\text { mindfulness }\end{array}$ & $\begin{array}{l}\text { Fases de } \\
\text { aplicación }\end{array}$ & $\begin{array}{l}\text { Áreas del } \\
\text { cerebro } \\
\text { asociadas }\end{array}$ \\
\hline $\begin{array}{l}\text { 1. Regulación de la } \\
\text { atención. }\end{array}$ & $\begin{array}{l}\text { Sostener la atención } \\
\text { en distintos puntos. }\end{array}$ & $\begin{array}{l}\text { Meditación de la } \\
\text { respiración. }\end{array}$ & $\begin{array}{l}\text { Fases iniciales/ } \\
\text { primeras sesiones. }\end{array}$ & $\begin{array}{l}\text { Córtex } \\
\text { cingulado } \\
\text { anterior. }\end{array}$ \\
\hline $\begin{array}{l}\text { 2. Conciencia } \\
\text { corporal. }\end{array}$ & $\begin{array}{l}\text { Sostener la atención } \\
\text { en las sensaciones } \\
\text { corporales: } \\
\text { respiración cuerpo, } \\
\text { emociones, etc. }\end{array}$ & $\begin{array}{l}\text { Meditación de la } \\
\text { contemplación de las } \\
\text { sensaciones o body scan. } \\
\text { Movimientos corporales } \\
\text { con atención plena } \\
\text { (mindful movements), etc. }\end{array}$ & $\begin{array}{l}\text { Fases iniciales e } \\
\text { intermedias. }\end{array}$ & $\begin{array}{l}\text { Ínsula, junta } \\
\text { temporo- } \\
\text { parietal. }\end{array}$ \\
\hline $\begin{array}{l}\text { 3a. Regulación } \\
\text { emocional: } \\
\text { revalorización. }\end{array}$ & $\begin{array}{l}\text { Generar nuevas } \\
\text { formas de reaccionar } \\
\text { a las emociones: no } \\
\text { juzgar, aceptar. }\end{array}$ & $\begin{array}{l}\text { Meditación de las } \\
\text { emociones. }\end{array}$ & $\begin{array}{l}\text { Fases o sesiones } \\
\text { intermedias y } \\
\text { finales. }\end{array}$ & $\begin{array}{l}\text { Córtex } \\
\text { prefontal } \\
\text { (dorsal). }\end{array}$ \\
\hline $\begin{array}{l}\text { 3b. Regulación } \\
\text { emocional: } \\
\text { exposición, } \\
\text { extinción y } \\
\text { reconsolidación. }\end{array}$ & $\begin{array}{l}\text { Exponerse a aquello } \\
\text { que sucede en la } \\
\text { conciencia: tomar } \\
\text { distancia, no } \\
\text { reaccionar a la } \\
\text { experiencia interna. }\end{array}$ & $\begin{array}{l}\text { Meditación de las } \\
\text { emociones. } \\
\text { Meditación de las } \\
\text { emociones difíciles. }\end{array}$ & $\begin{array}{l}\text { Fases o sesiones } \\
\text { intermedias y } \\
\text { finales. }\end{array}$ & $\begin{array}{l}\text { Amígdala. } \\
\text { Hipocampo. }\end{array}$ \\
\hline $\begin{array}{l}\text { 4. Cambios en la } \\
\text { perspectiva del self. }\end{array}$ & $\begin{array}{l}\text { Desapego a una } \\
\text { imagen fija de uno } \\
\text { mismo. }\end{array}$ & $\begin{array}{l}\text { Meditación de los } \\
\text { pensamientos. } \\
\text { Meditación de la } \\
\text { disolución del yo. }\end{array}$ & $\begin{array}{l}\text { Transversal a } \\
\text { todo el } \\
\text { aprendizaje. } \\
\text { Mayor énfasis en } \\
\text { fases intermedias } \\
\text { y finales. }\end{array}$ & $\begin{array}{l}\text { Córtex } \\
\text { cingulado } \\
\text { posterior, } \\
\text { ínsula. }\end{array}$ \\
\hline
\end{tabular}

El primero de los mecanismos es el de control atencional. Este es el primer nivel de interacción en la práctica (cuando llevamos la atención al aire entrando o 
saliendo de la nariz) y, de hecho, supone la primera dificultad en su práctica (divagación mental, dificultades para retener la atención en un punto). Sabemos a través de tareas experimentales que la atención mejora de manera clara tras un entrenamiento protocolizado de mindfulness (Tang, Hölzel, \& Posner, 2015), y de hecho el esfuerzo atencional es muy alto al inicio del entrenamiento y se va reduciendo conforme aumenta el número de horas de práctica (Tang, Yang, Leve, \& Harold, 2012). Esta mejora en los niveles atencionales es en la que se basa la justificación del uso de mindfulness en el ámbito escolar o en el tratamiento del TDAH (van de Weijer-Bergsma, Formsma, de Bruin, \& Bögels, 2011) o del deterioro cognitivo adulto (Quintana-Hernández et al., 2015).

En esta primera fase la instrucción básica que guiará las distintas prácticas utilizadas hará énfasis en llevar la atención a la respiración o al cuerpo y mantenerla hasta que inevitablemente esta se aleje, y será entonces cuando debamos hacer que regrese al foco de atención de la práctica (por ejemplo a la respiración o al cuerpo) con una actitud de amabilidad. Este "ir y venir" supone el punto de partida y el núcleo de la práctica en los entrenamientos de mindfulness.

Distintas tradiciones meditativas enfatizan la importancia de la regulación atencional en la práctica diaria, por ejemplo, samadhi en la tradición Budista Theravada (Hart, 1987); samatha en la tradición budista Tibetana (Lutz, Dunne, \& Davidson, 2007); o dharana en la tradición de Yoga India (Vishnu Devananda, 1999). Se recomienda la meditación de atención focalizada como un primer nivel de aprendizaje antes de pasar a otro tipo de meditaciones. En las primeras sesiones de las terapias basadas en mindfulness (TBM) tanto el contenido de las mismas como las prácticas entre sesiones se destinarán a este fin. Es fundamental un buen entrenamiento antes de avanzar en el aprendizaje ya que supondrá un anclaje donde se volverá repetidamente. En resumen, además de ser un mecanismo subyacente a la práctica vemos que también es esencial en la propia práctica de mindfulness. En algunos casos puede ser una fuente de frustración y un obstáculo en el aprendizaje, por lo que se recomienda que el terapeuta/instructor esté atento y dedique recursos a establecer este primer nivel de práctica.

El segundo mecanismo es de conciencia corporal; el cuerpo surge en mindfulness como un espacio de observación de la experiencia. La observación momento a momento se hace sobre la experiencia que surge en el cuerpo y tanto la respiración como las sensaciones se utilizan como anclaje para regresar al aquí y ahora. Durante la práctica el cuerpo está presente siempre y es un lugar de observación neutral para depositar la atención, ya sea como el fenómeno físico de la respiración o el impacto de los pensamientos o emociones en el cuerpo. Para ello se pueden utilizar prácticas que están directamente dirigidas a este propósito, como el body scan u otras relacionadas en las que se incluye la instrucción básica de la conciencia corporal, como por ejemplo la meditación de la respiración (prestar atención a las sensaciones físicas y cambios corporales de la respiración).

Por conciencia corporal se entiende un proceso dinámico e interactivo por el 
que se perciben los estados, procesos, y acciones que suceden en el cuerpo tanto a nivel interoceptivo como propioceptivo y que pueden ser observados por uno mismo (Mehling et al., 2005). Este mecanismo está muy conectado con las teorías de embodiment que parten de la base de que la cognición se basa en representaciones de las distintas modalidades sensoriales (visual, auditiva, táctil y cenestésica).

Según esta aproximación los estados corporales son constituyentes de la cognición y, por lo tanto, pueden desencadenar estados cognitivos y viceversa (Barsalou, Niedenthal, Barbey, \& Ruppert, 2003). La postura corporal, los movimientos de expansión o los ejercicios de yoga se utilizan de manera consciente para trabajar este aspecto clave. Los datos indican que tras un entrenamiento en mindfulness aumenta la conciencia corporal y también la agencia del cuerpo (Cebolla, Vara, Miragall, Palomo y Baños, 2015).

Por otro lado, nuevas hipótesis plantean que este mecanismo está profundamente conectado con todos los demás y sobre todo con el último, el cambio en la perspectiva del self ya que altera los procesos de la auto-representación. Desde un punto de vista pedagógico, el cuerpo representa el "lugar" donde ocurren nuestras experiencias y sirve como espacio de observación, una observación adaptativa, basada en el presente y no juzgadora (Michalak, Burg, \& Heidenreich, 2012). En términos metafóricos sería el "laboratorio" o el espacio de trabajo. Es ahí donde el terapeuta/instructor invita a mirar (Segal, Williams, \& Teasdale, 2013) cuando algo nos invita a salir de la práctica o aparecen pensamientos o emociones difíciles. Esta sería la posición del instructor y el papel que tomará como hilo conductor en el proceso de enseñanza.

El tercer y cuarto mecanismo son en realidad dos formas de entender la regulación emocional. En los últimos años han surgido una variedad de trabajos que sugieren que la práctica de mindfulness facilita la regulación emocional (Hölzel et al., 2011). Por regulación emocional se entiende el conjunto de procesos a través de los cuales influimos en las emociones que tenemos, cuándo las tenemos y cómo las experimentamos y expresamos (Gross, 1998). Concretamente desde las TBM, se proponen dos estrategias de regulación emocional como son la re-valoración y la exposición.

La re-valoración hace referencia al proceso de adaptación a través del cual los eventos estresantes son reconstruidos como beneficiosos, significativos o benignos (por ejemplo, pensar que uno va a aprender algo de una situación difícil; Garland, Gaylord, \& Fredrickson, 2011). Además, durante la meditación nos exponemos a todo lo que está presente en el campo de la conciencia, incluyendo estímulos externos así como sensaciones corporales y experiencias emocionales, observando estas experiencias sin alterarlas o reaccionar a ellas. Por tanto, atender a las emociones o sensaciones desagradables desde esta actitud genera a largo plazo una mayor capacidad de regularlas.

En este punto del aprendizaje las instrucciones básicas radicarán en facilitar estas dos estrategias de regulación. Para la revalorización nos centraremos en 
generar nuevas formas de reaccionar a las emociones a través de "no juzgar" y aceptar la experiencia, mientras que para facilitar la exposición, extinción y reconsolidación fomentaremos el tomar distancia de las experiencias que sucedan en la consciencia, observando sin reaccionar a la experiencia interna. Aunque todavía quedan muchos aspectos por clarificar de la relación entre mindfulness y regulación emocional, estas son algunas ideas que nos pueden ser de utilidad en la pedagogía de mindfulness, sobre todo en fases intermedias cuando se integra este componente.

En el cuarto nivel, se profundiza en los cambios en la perspectiva del self, como consecuencia de lo trabajado previamente. Se trata de poner la atención en la naturaleza transitoria del self. Es lo que se conoce como la "desconstrucción de uno mismo" (Epstein, 1988) y aparece con la experiencia de la práctica continuada. Cuando aumenta la conciencia interna a través del entrenamiento los meditadores informan de una mayor capacidad y claridad para observar los procesos mentales (MacLean et al., 2010). Esta mayor claridad facilita la observación de cómo los procesos mentales aparecen y desaparecen, consolidando la idea de que el contenido mental es transitorio y está en constante cambio tal y como sugieren Hölzel et al. (2011).

Además, la observación sin juzgar que se enseña y entrena en mindfulness permite el desapego o desidentificación de los contenidos de la conciencia. Es lo que algunos autores, llaman "repercepción" o "descentramiento" (Carmody, Baer, Lykins, \& Olendzki, 2009; Fresco et al., 2007; Shapiro, Carlson, Astin, \& Freedman, 2006). En estas fases se habla de la perspectiva del observador: el "yo observador" diferente del observado. De esta forma, se genera el desapego de las experiencias. Desapego se refiere a la ausencia de una fijación de ideas, imágenes u objetos sensoriales así como la ausencia de presión interna para mantener, evitar o cambiar las experiencias (Shadra, Shaver, \& Brown, 2010). De acuerdo con las tradiciones budistas, este cambio en la perspectiva del self es la clave para la felicidad.

Tal y como se observa en la Figura 1 estos mecanismos siguen un orden y cada uno es esencial para explicar los otros. El control atencional se lleva a cabo sobre todo sobre la conciencia corporal y es observando esta conciencia cuando surgen las emociones y el trabajo de regulación de las mismas a través de la atención y el cuerpo lleva a modificar la perspectiva del yo, generando una perspectiva metacognitiva y descentrada.

En los protocolos de las TBM se utilizan metáforas para representar y fomentar este fenómeno, como sería la del espectador de cine en el que uno puede ver en la pantalla todo lo que aparece y desaparece (pensamientos, emociones, etc.) como algo que se observa y no es uno mismo. En este punto del aprendizaje la instrucción básica enfatiza el desapego a una imagen fija de uno mismo, haciendo consciente al practicante de esta evidencia durante la práctica así como en el proceso de indagación que más adelante comentaremos.

Algunos autores plantean que este proceso de meta-conciencia, es decir, de darse cuenta de la propia conciencia, es una forma de experiencia subjetiva y control 


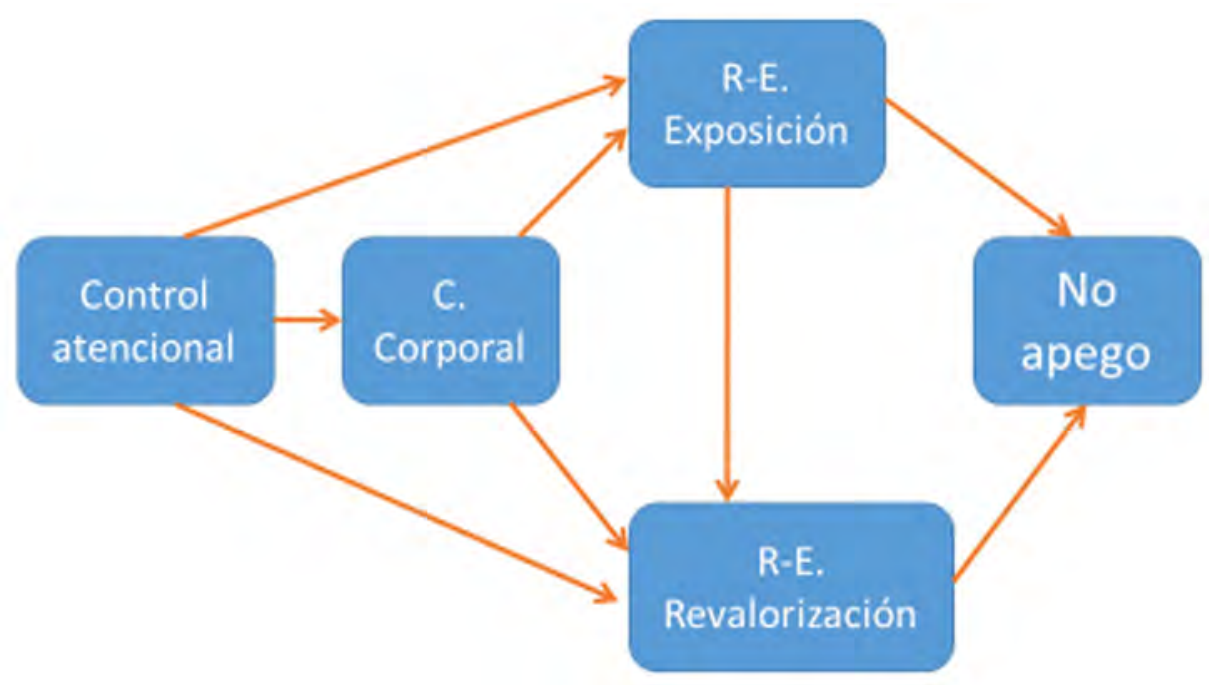

Figura 1

Modelo Pedagógico Basado en los Mecanismos de Eficacia

ejecutivo en que uno adopta una perspectiva no-conceptual como una forma de atención hacia los contenidos de la conciencia y los procesos implicados (Deikman, 1982; Raffone, \& Pantani, 2010; Varela, Thompson, \& Rosch, 1991). Este concepto es similar al insight metacognitivo (Teasdale et al., 1995) que se persigue desde la MBCT y que promueve la observación de los pensamientos como pensamientos y no como descripciones de la realidad, con una actitud no enjuiciadora de apertura, curiosidad y aceptación. Estos elementos son claves en el proceso de enseñanza y jugarán un papel esencial en el proceso de indagación. Por tanto, el terapeuta/ instructor tratará de fomentarlos a lo largo de todo el proceso. Es más, buscará el insight metacognitivo como una competencia transversal a lo largo del proceso de enseñanza.

Para resumir, tal como hemos mencionado anteriormente la investigación sobre la pedagogía de los mecanismos subyacentes a la eficacia de mindfulness puede ayudar a generar un modelo pedagógico de mindfulness basado en la evidencia que sea flexible y adaptable a las características de cada persona. Con la idea de facilitar este proceso, hemos resumido los principales mecanismos organizándolos secuencialmente desde las primeras fases a fases más avanzadas del aprendizaje.

A partir del modelo de Hölzel et al. (2011) establecemos los distintos mecanismos implicados y vemos cómo se relacionan con la práctica y la enseñanza de mindfulness en las distintas fases del aprendizaje. Pueden ser una guía a la hora de enseñar mindfulness para que el terapeuta/instructor centre su atención en los puntos importantes a recalcar en cada sesión, según en el punto de la enseñanza en el que se encuentre. 


\section{La Pedagogía de Mindfulness}

Mindfulness es un constructo de muy compleja comprensión si únicamente se explica en términos conceptuales, es decir sin atender a la experiencia directa. De hecho, en un estudio donde se comparó una formación experiencial (basada en la práctica) en la enseñanza de mindfulness con una formación estrictamente teórica (sin práctica) se observó una gran diferencia en la comprensión y en el número e intensidad de los obstáculos que surgen en la práctica (Cebolla y García-Palacios, 2013). De cara a ejemplificar una propuesta pedagógica para la enseñanza de mindfulness Segal, Williams, \& Teasdale (2013) utilizan el modelo pedagógico de Kolb (1984; véase Figura 2).

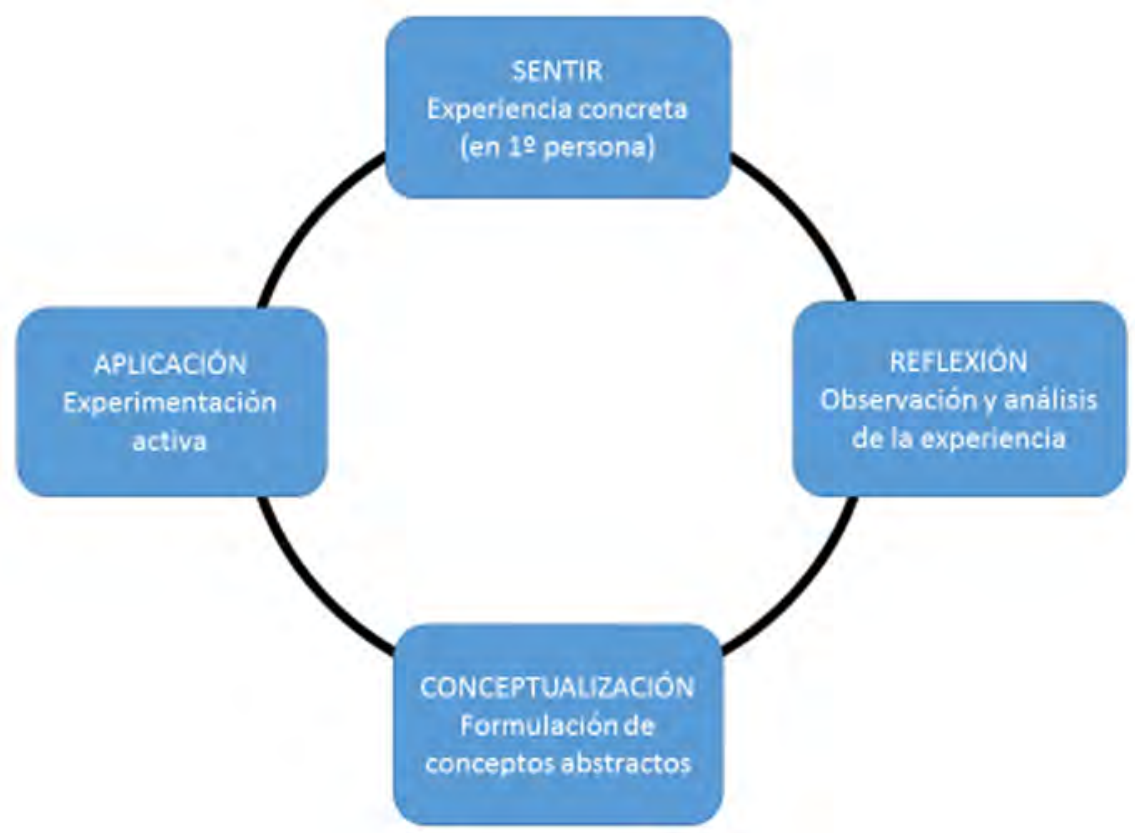

\section{Figura 2}

\section{El Modelo de Kolb (1984)}

El punto de partida siempre es la experiencia directa y en primera persona. Posteriormente se pasa a la reflexión, en la que se atiende al contenido de la experiencia de forma descriptiva (poner en palabras experiencia directa). La tercera fase se centra en dar un sentido a la experiencia vivida y relacionarla con cuestiones más amplias como el contexto. Finalmente el último eslabón se dedica a las implicaciones que tiene el aprendizaje en la vida y en futuras experiencias.

Partiendo del modelo de Kolb (1984) en el que el foco del aprendizaje está en la experiencia directa se estructura lo que se ha llamado el proceso de indagación. Este proceso es el elemento pedagógico más importante en el proceso de transmi- 
sión y la esencia de las dinámicas grupales basadas en mindfulness. Requiere que el terapeuta/instructor acentúe su habilidad para escuchar atentamente, dejar espacio, frenar el impulso de dar consejos y, en lugar de eso, investigue directamente en la experiencia real del participante.

El objetivo (Williams et al., 2007) es sonsacar a los participantes lo notado durante la práctica $\mathrm{y}$, al hacerlo, animarlos a que reflexionen sobre su experiencia y guiarlos sobre cómo explorarla. Por otro lado genera el espacio para trabajar juntos a través del diálogo sobre estas observaciones, para vincular estas observaciones y descubrimientos con los objetivos del programa de intervención. El proceso de indagación es una disciplina de improvisación centrada en las condiciones específicas del momento en el que tiene lugar, caracterizada por una serie de patrones repetitivos e identificables de interacción. Concretamente los autores describen y analizan tres prácticas del proceso de indagación que se pueden identificar en las secuencias del discurso. Estas son:

1. Turnos de conversación implicando preguntas y reformulaciones.

2. El desarrollo de las habilidades de los participantes mediante una forma particular de describir la experiencia.

3. El discurso que construye las conexiones intersubjetivas y la afiliación dentro del grupo.

Los turnos de conversación se refieren a un proceso de co-construcción entre el instructor y el/los participante/s compuesto por tres secuencias o turnos de habla (Crane et al., 2014; Lee, 2007). En primer lugar el instructor abre el diálogo (Primer Turno) (por ejemplo preguntando lo sucedido tras un práctica de mindfulness) a lo que siguen las respuesta del/los participante/s en las que se reconstruyen conjuntamente las descripciones de las experiencias (Segundo Turno) y, en última instancia, el instructor da respuesta, recopilando, reformulando y expandiendo el aprendizaje a todo el grupo (Tercer Turno). En este sentido, Crane et al. (2014) observaron a través del análisis conversacional que los instructores utilizaban varias metodologías y respuestas en los turnos Primero y Tercero, teniendo como punto común la reformulación del discurso de los participantes para cumplir los objetivos del entrenamiento en mindfulness.

El desarrollo de las habilidades de los participantes mediante una forma particular de describir la experiencia se refiere a que en el proceso de indagación el instructor va guiando y configurando el discurso de los participantes hacia las normas de conversación características de las TBM. Crane et al. (2014) observan distintos métodos a través de los cuales el instructor busca entrenar las competencias de los participantes para describir sus experiencias de forma consistente con la práctica de mindfulness.

En primer lugar los participantes aprenden a anclar su aprendizaje en la experiencia directa más que en ideas generalizadas sobre ésta (primera persona). En este punto el instructor redirigirá el discurso de los participantes cuando detecte que se vuelve menos específico. 
En segundo lugar tratará de generalizar la experiencia de los participantes que suelen describir sus experiencias en primera persona utilizando el pronombre "yo". Por eso en el tercer turno el instructor hace uso de términos como "nuestro" o "nosotros" además de hablar sobre "la" mente en vez de "tu" mente. De esta forma se extraen los temas que pueden ser comunes a todos los miembros del grupo, facilitando la generalización el aprendizaje.

En tercer lugar el instructor muestra mayor interés dedicando más tiempo al turno de palabra del participante cuando su discurso es detallado, rico, no-analítico y sobre una experiencia inmediata referida a un tema clave de aprendizaje. Por el contrario, cuando las contribuciones del participante no fomentan esto, el instructor redirige el discurso explícitamente o utiliza respuestas cortas para finalizar el turno.

En cuarto lugar el instructor fomenta el interés y la curiosidad por la experiencia cotidiana así como por los patrones mentales que previamente pasaban desapercibidos. Con este objetivo el instructor anima activamente a los participantes a atender y reconocer que en este contexto lo que puede parecer irrelevante $u$ ordinario cobra especial relevancia.

En quinto lugar los instructores fomentan en los participantes el uso de términos como "darse cuenta" o "ser consciente de" para co-construir retrospectivamente sus experiencias a través del lenguaje. Crane et al. (2014) observaron que los instructores incluso recompensaban estas habilidades de los participantes enfatizándolas a través de evaluaciones positivas como un darse cuenta positivo.

El discurso que construye las conexiones intersubjetivas y la afiliación dentro del grupo. Los instructores a menudo utilizan estrategias durante los turnos de conversación para asegurar la participación de los miembros del grupo y fomentar la afiliación, reconociendo que el tema que se está explorando es relevante para todos. Además el instructor, a través del discurso y la práctica repetida, va construyendo conexiones con las experiencias que van narrando los participantes y el sentido de universalidad de la experiencia humana. Otras expresiones utilizadas son las respuestas cortas altamente positivas como "sí" o "mmm", que expresan el cierre y cambio de tema. Estos recursos pueden ser de utilidad para marcar el ritmo del turno de habla y gestionar el grupo.

En este sentido, Segal, Williams y Teasdale (2013) hacen una propuesta muy útil y pedagógica similar compuesta por tres círculos o estratos del proceso de indagación $\mathrm{y}$, al igual que la anterior, la dividen en tres círculos o estratos concéntricos.

El primero, tal y como se ha indicado en el modelo de Kolb, es el de la experiencia directa, el conocimiento directo en primera persona y experiencial de los fenómenos que han surgido en la práctica de mindfulness. En este proceso las preguntas del instructor que enseña mindfulness deben ir dirigidas a lo notado en el cuerpo (por ejemplo, sensaciones físicas, emociones/sentimiento), en la mente (por ejemplo, pensamientos, imágenes), fuera del cuerpo (por ejemplo, sonidos) y, por último, el contenido de la distracción (pensamientos, sensaciones o emociones). 
Una vez el participante ha podido describir (no analizar ni juzgar) en primera persona lo experimentado se pasaría a la segunda fase o círculo. Es en este círculo donde los mecanismos de regulación de la atención, conciencia corporal y regulación emocional del modelo de Hölzel et al. (2011) indicados previamente cobran todo su sentido.

La segunda fase del proceso de indagación (segundo círculo o estrato) está más centrada en el proceso de darse cuenta, es decir el foco atencional es la observación del contenido mental desde una perspectiva descentrada. En este punto se estimulará por parte del instructor un yo observador consciente del yo observado. Se estimula a partir de preguntas como, ¿cómo te sentiste cuando te diste cuenta de... (por ejemplo que tu mente divagaba, que una emoción de frustración ha aparecido, etc.)?, ¿qué hiciste cuando te diste cuenta de... (por ejemplo que te habias distraído, que tenías una sensación desagradable, etc.)? En este círculo es esencial establecer una actitud de auto-observación adaptativa (basada en el presente, amable, no juzgadora) (Watkins, \& Teasdale, 2004) hacia la experiencia interna.

Finalmente se avanza hacia el último estrato o círculo externo cuyo objetivo es la integración. En esta fase el instructor debe tener un buen entrenamiento ya que se trata de tejer un diálogo sobre la experiencia directa y los efectos que tiene poner una atención consciente sobre esta experiencia. Es decir, vincular lo vivido con el modelo de funcionamiento psicológico basado en mindfulness que se pretende mostrar (en el caso de aplicar un protocolo, emplear las propuestas del modelo), por el que la observación directa y amable de la experiencia tiene un potencial transformador. Esta comprensión estaría a la base del mecanismo de mindfulness de la perspectiva del self y se parecería mucho a lo que Teasdale situó como la auténtica esencia de un entrenamiento en mindfulness, lograr el insight metacognitivo (Teasdale, Segal, \& Williams, 1995). Cada uno de estos estratos o fases fundamenta el siguiente estrato; además no son estratos cerrados y excluyentes sino un continuo en el que se va tejiendo un dialogo.

En resumen, el proceso de indagación tiene una dirección y un propósito particular alineado con los objetivos de las TBMs; el instructor establece las normas del contenido que se incluye y excluye en este proceso basado en el marco de referencia de interacción, métodos y estructuras que permiten el diálogo (Crane et al., 2014). No obstante, este es un proceso de improvisación en el que el instructor responde al proceso y modifica sus métodos para lograr los objetivos. Desde este planteamiento no se busca una respuesta correcta específica ni cambiar la opinión de la persona que aprende o practica mindfulness sino guiarlo en el descubrimiento.

Por estos motivos una habilidad de suma importancia que debe tener el instructor es la capacidad de "bailar" con la demanda de cada momento mientras se conduce el proceso de aprendizaje, lo cual nos conecta con las competencias necesarias por parte de los instructores para la enseñanza de mindfulness (Segal, Williams, \& Teasdale, 2013), como son la curiosidad e interés genuino, la amabilidad y la validación de la experiencia, corporeizar la experiencia vivida (no 
sólo moderar la de los otros), no pretender arreglar las cosas, generar un espacio de diálogo, ser respetuoso con los tiempos, tener capacidad de improvisar y formular preguntas abiertas que eviten respuestas rápidas.

\section{Nuevos Retos}

El estudio de la pedagogía de mindfulness tiene muchos retos por delante y necesita que investigaciones específicas profundicen en las estrategias que faciliten en mayor medida el aprendizaje y la utilidad de la herramienta de mindfulness. Uno de los retos más relevantes es la incorporación de otras prácticas contemplativas, como la compasión o las meditaciones analíticas, que suponen un planteamiento y un posicionamiento diferente al de mindfulness.

Por otro lado el uso de las tecnologías de la información y la comunicación, tales como aplicaciones móviles o programas on-line, para la formación en mindfulness también son un reto importante para poder ejemplificar el objetivo de un entrenamiento en mindfulness. Otro reto es el de la comunicación entre ciencia y tradiciones contemplativas: mindfulness es sólo una de las muchas estrategias que se han formulado desde las tradiciones espirituales. Tal y como proponen Dhal, Lutz y Davidson (2015) existen tres grandes familias de prácticas contemplativas: las atencionales (atención a la respiración), las constructivas (compasión, valores, etc.) y la desconstructivas (mindfulness) y cada una de ellas requerirá una propuesta pedagógica diferente adaptada a nuevos tiempos y nuevas demandas.

\section{Conclusiones}

Las formas de enseñar y aprender han trasformado el contexto de enseñanza donde tradicionalmente se ha enseñado mindfulness suponiendo un gran reto por parte de la psicología académica.

A pesar de que se ha hecho mucha investigación de resultados, apenas se ha publicado sobre investigación de procesos, narrativas y relación terapéutica en el ámbito de la enseñanza de mindfulness. Este elemento normalmente es minimizado por parte de algunas formaciones, delegando todo el aprendizaje a un proceso natural consecuencia directa de la práctica o del insight.

Sin duda alguna, la enseñanza de mindfulness no debe reducirse a un proceso guiado por la intuición, sino que debe basarse en modelos científicos de enseñanza y aprendizaje que optimicen el aprendizaje y minimicen los abandonos. Lo que todo instructor que aplica mindfulness debe evitar a toda costa es que haya alguien que pueda interpretar que no tiene las características necesarias para aprender y practicar mindfulness. Éste sin duda sería un fracaso de la pedagogía, en ningún caso del practicante.

Cabe remarcar que en este artículo no hemos centrado nuestra atención en los contenidos a impartir sino en los procesos en los que intervenimos cuando enseñamos mindfulness. En la actualidad contamos con manuales estandarizadas que podemos utilizar como guía para establecer qué contenidos trabajar e impartir, 
no obstante hemos querido reflexionar sobre la pedagogía de mindfulness tratando de clarificar qué estamos enseñando y cómo podemos fomentar o facilitar este aprendizaje.

\section{Referencias bibliográficas}

Barsalou, L. W., Niedenthal, P. M., Barbey, A. K., \& Ruppert, J. A. (2003). Social embodiment. Psychology of Learning and Motivation, 43, 43-92.

Carmody, J., Baer, R.A., Lykins, E. L. B., \& Olendzki, N. (2009). An empirical study of the mechanisms of mindfulness in a mindfulness based stress reduction program. Journal of Clinical Psychology, 65, 613-626.

Cebolla, A. (2014). ¿Por qué y para qué sirve practicar mindfulness? Mecanismos de acción y eficacia. En A. Cebolla, J. Garciì-Campayo y M. Demarzo (Coords). Mindfulness y Ciencia. De la Tradición a la Modernidad ( $1^{\text {a }}$ Ed., pp. 65-84). Madrid: Alianza Editorial.

Cebolla, A., Demarzo, M., Martins P., Soler, J., y Garcia-Campayo, J. (en revisión). Unwanted effects: is there a negative side of meditation? A multicentre survey. Material no publicado.

Cebolla, A., y Miró, M.T. (2006). Eficacia de la terapia cognitiva basada en la atención plena en el tratamiento de la depresión. Revista de Psicoterapia, 66/67, 133-156. http://revistadepsicoterapia.com/eficacia-de-laterapia-cognitiva-basada-en-la-atencion-plena-en-el-tratamiento-de-la-depresion.html

Cebolla, A., Vara, MD., Miragall, M., Palomo, P., y Baños, R. (2015) Embodied mindfulness: review of the body's participation in the changes associated with the practice of mindfulness. Actas Españolas de Psiquiatria, 43, 36-41.

Cebolla, A., y García-Palacios, A. (2013). Efectos de una aproximación experiencial en la adquisición de competencias asociadas al aprendizaje de la técnica de mindfulness dentro de la asignatura de técnicas de intervención y tratamientos psicológicos. En A. Bengochea (dir.), IV Jornada Nacional sobre Estudios Universitarios: El futuro de los títulos universitarios (pp.190-197). Castellón de la Plana, España: Publicacions de la Universitat Jaume I.

Crane, R. S., Stanley, S., Rooney, M., Bartley, T., Cooper, L., \& Mardula, J. (2015). Disciplined improvisation: Characteristics of inquiry in mindfulness-based teaching. Mindfulness, 6(5), 1104-1114.

Dahl, C. J., Lutz, A., \& Davidson, R. J. (2015). Reconstructing and deconstructing the self: cognitive mechanisms in meditation practice. Trends in Cognitive Sciences, 19(9), 515-523.

Deikman, A. J. (1982). The observing self: Mysticism and psychotherapy. Boston, MA: Beacon Press.

Epstein, M. (1988). The deconstruction of the self: Ego and "egolessness" in Buddhist Insight meditation. The Journal of Transpersonal Psychology, 20, 61-69.

Fresco, D. M., Moore, M. T., van Dulmen, M. H., Segal, Z. V., Ma, S. H., Teasdale, J. D., \& Williams, J. M. (2007). Initial psychometric properties of the experiences questionnaire: Validation of a self-report measure of decentering. Behavior Therapy, 38, 234-246.

García, A. (2006). Mindfulness en la terapia dialéctico-comportamental. Revista de Psicoterapia, 66/67, $121-132$. http://revistadepsicoterapia.com/mindfulness-en-la-terapia-dialectico-comportamental.html

Garland, E. L., Gaylord, S. A., y Fredrickson, B. L. (2011). Positive reappraisal mediates the stress-reductive effects of mindfulness: An upward spiral process. Mindfulness, 2, 59-67

Gross, J. J. (1998). The emerging field of emotion regulation: An integrative review. Review of General Psychology, 2, 271-299.

Grossman, P. (2015). Mindfulness: Awareness informed by an embodied ethic. Mindfulness, 6(1), 17-22.

Hart, W. (1987). The art of living: Vipassana meditation: As taught by S.N. Goenka. San Francisco, CA: HarperOne.

Hölzel, B. K., Lazar, S. W., Gard, T., Schuman-Olivier, Z., Vago, D. R., \& Ott, U. (2011). How does mindfulness meditation work? Proposing mechanisms of action from a conceptual and neural perspective. Perspectives on Psychological Science, 6(6), 537-559.

Kolb, D. A. (1984). Experiental learning: experience as the source oflearning and development. Englewood Cliffs, NJ: Prentice Hall.

Kuyken, W., Hayes, R., Barrett, B., Byng, R., Dalgleish, T., Kessler, D., Lewis, G., Watkins, E., Brejcha, C., Cardy. J., Causley, A., Cowderoy, S., Evans, A., Gradinger, F., Kaur, S., Lanham, P., Morant, N., Richards, J., Snah, P., Sutton, H., Vicary, R., Weaver, A, Wilks, J., Williams, M., Taylor, R. S., \& Byford, S. (2015). Effectiveness and cost-effectiveness of mindfulness-based cognitive therapy compared with maintenance antidepressant treatment in the prevention of depressive relapse or recurrence (PREVENT): a randomised controlled trial. The Lancet, 386(9988), 63-73. 
Lee, Y. O. (2007). Third turn position in teacher talk: contingency and the work of teaching. Journal of Pragmatics, 39, 180-206.

Lutz, A., Dunne, J. D., \& Davidson, R. J. (2007). Meditation and the neuroscience of consciousness: An introduction. In P.D. Zelazo, M. Moscovitch, \& E. Thompson(Eds.), Cambridge handbook of consciousness (pp. 499-554). Cambridge: Cambridge University Press.

MacLean, K.A., Ferrer, E., Aichele, S.R., Bridwell, D.A., Zanesco, A.P., Jacobs, T.L., King, B.G., Rosenberg, E.L., Sahdra, B.K., Shaver, P.R., Wallace, B.A., Mangun, G.R., \& Saron, C.D. (2010). Intensive meditation training improves perceptual discrimination and sustained attention. Psychological Science, 21, 829-839.

Mehling, W.E., Gopisetty, V., Daubenmier, J., Price, C.J., Hecht, F.M., \& Stewart, A. (2009). Body awareness: Construct and self report measures. PLoS ONE, 4, e5614.

Michalak, J., Burg, J., \& Heidenreich, T. (2012). Don't forget your body: Mindfulness, embodiment, and the treatment of depression. Mindfulness, 3(3), 190-199.

Miró, M. T. (2006). La atención plena (mindfulness) como intervención clínica para aliviar el sufrimiento y mejorar la convivencia. Revista de Psicoterapia, 66/67, 31-76. http://revistadepsicoterapia.com/la-atencion-plenamindfulness-como-intervencion-clinica-para-aliviar-el-sufrimiento-y-mejorar-la-convivencia.html

Pérez, M. A., y Botella, L. (2006). Conciencia plena (mindfulness) y psicoterapia: Concepto, evaluación y aplicaciones clínicas. Revista de Psicoterapia, 66/67, 77-120. http://revistadepsicoterapia.com/concienciaplena-mindfulness-y-psicoterapia-concepto-evaluacion-y-aplicaciones-clinicas.html

Quintana-Hernández, D. J., Miró-Barrachina, M. T., Ibáñez-Fernández, I. J., Pino, A. S. D., Quintana-Montesdeoca, M. P., Rodríguez-de Vera, B., Morales-Casanova, D., Perez-Vieitez, M., Rodriguez-García, J,\& BravoCaraduje, N. (2015). Mindfulness in the maintenance of cognitive capacities in Alzheimer's disease: a randomized clinical trial. Journal of Alzheimer's Disease, 50(1), 1-16.

Raffone, A., \& Pantani, M. (2010). A global workspace model for phenomenal and access consciousness. Consciousness and Cognition, 19, 580-596.

Sahdra, B. K., Shaver, P. R., \& Brown, K. W. (2010) A scale to measure nonattachment: A Buddhist complement to Western research on attachment and adaptive functioning. Journal of Personality Assessment, 92, 1-12, 2010 .

Santamaría, M. T., Cebolla, A., Rodríguez, P. J. y Miró, M. T. (2006). La práctica de la meditación y la atención plena: Técnicas milenarias para padres del siglo XXI. Revista de Psicoterapia, 66/67, 157-175. http:// revistadepsicoterapia.com/la-practica-de-la-meditacion-y-la-atencion-plena-tecnicas-milenarias-para-padres-del-siglo-xxi.html

Segal Z. V., Williams J. M. G., \& Teasdale J. D. (2013). Mindfulness-based cognitive therapy for depression. 2nd ed. New York: Guilford Publications.

Shapiro, D.H. (1992) Adverse effects of Meditation: A Preliminary Investigation of long-term meditators. International Journal of Psychosomatics, 39, 62-67.

Shapiro, S. L., Carlson, L. E., Astin, J. A., y Freedman, B. (2006). Mechanisms of mindfulness. Journal of Clinical Psychology, 62, 373-386.

Simón, V. (2006). Mindfulness y neurobiología. Revista de Psicoterapia, 66/67, 5-30. http:// revistadepsicoterapia.com/mindfulness-y-neurobiologia.html

Tang, Y. Y., Hölzel, B. K., \& Posner, M. I. (2015). The neuroscience of mindfulness meditation. Nature Reviews Neuroscience, 16(4), 213-225.

Tang, Y. Y., Yang, L., Leve, L. D., \& Harold, G. T. (2012). Improving executive function and its neurobiological mechanisms through a mindfulnes-based intervention: Advances within the field of developmental neuroscience. Child development perspectives, 6(4), 361-366.

Teasdale, J. D., Segal, Z., \& Williams, J. M. G. (1995). How does cognitive therapy prevent depressive relapse and why should attentional control (mindfulness) training help? Behaviour Research and Therapy, 33(1), 25-39.

van de Weijer-Bergsma, E., Formsma, A., de Bruin, E., \& Bögels, S. (2011). The effectiveness of mindfulness training on behavioral problems and attentional functioning in adolescents with ADHD. Journal of Child and Family Studies, 21, 775-787.

Varela, F.J., Thompson, E., \& Rosch, E. (1991). The embodied mind. Cambridge, MA: MIT Press.

Vishnu Devananda, S. (1999). Meditation and mantras. Delhi: Motilal Banarsidass Publishers.

Watkins, E., \& Teasdale, J. D. (2004). Adaptive and maladaptive self-focus in depression. Journal of affective disorders, 82(1), 1-8.

Williams, M.G., Crane, C., Soulsby, J.M., Blacker, M., Meleo-Meyer, F., Stahl (2007) The inquiry process, aims, intentions and teaching considerations. Manuscrito no publicado. 